\title{
Time delay in the Kuramoto model with bimodal frequency distribution
}

\author{
Ernest Montbrió, ${ }^{1,2,3}$ Diego Pazó,,* and Jürgen Schmidt ${ }^{5}$ \\ ${ }^{1}$ Computational Neuroscience, Technology Department, Universitat Pompeu Fabra, 08003 Barcelona, Spain \\ ${ }^{2}$ Departament de Física, Universitat Illes Balears, 07122 Palma de Mallorca, Spain \\ ${ }^{3}$ Institut Mediterrani d'Estudis Avançats IMEDEA (CSIC-UIB), 07122 Palma de Mallorca, Spain \\ ${ }^{4}$ Max-Planck-Institut für Physik komplexer Systeme, Nöthnitzer Straße 38, 01187 Dresden, Germany \\ ${ }^{5}$ Nichtlineare Dynamik, Institut für Physik, Universität Potsdam, 14415 Potsdam, Germany \\ (Received 15 June 2006; revised manuscript received 9 August 2006; published 2 November 2006)
}

\begin{abstract}
We investigate the effects of a time-delayed all-to-all coupling scheme in a large population of oscillators with natural frequencies following a bimodal distribution. The regions of parameter space corresponding to synchronized and incoherent solutions are obtained both numerically and analytically for particular frequency distributions. In particular, we find that bimodality introduces a new time scale that results in a quasiperiodic disposition of the regions of incoherence.
\end{abstract}

DOI: 10.1103/PhysRevE.74.056201

PACS number(s): 05.45.Xt, 89.75.Fb, 02.30.Ks

\section{INTRODUCTION}

The Kuramoto model [1] is presumably the most successful attempt to study macroscopic synchronization phenomena arising in large heterogeneous populations of interacting self-oscillatory units [2-4]. Kuramoto, motivated by Winfree's work on biological oscillators [5], showed that the dynamics of an ensemble of $N$ weakly interacting limit-cycle oscillators can be treated considering simply the oscillator phases $\left(\theta_{1}, \ldots, \theta_{N}\right)$. In this paper, we study the Kuramoto model with delayed interactions [6],

$$
\dot{\theta}_{i}(t)=\omega_{i}-\frac{K}{N} \sum_{j=1}^{N} \sin \left[\theta_{i}(t)-\theta_{j}(t-\tau)\right]+\xi_{i}(t), \quad i=1, \ldots, N,
$$

where heterogeneity is established considering a certain distribution $g(\omega)$ of the natural frequencies $\omega_{i}$. The terms $\xi_{i}(t)$ represent uncorrelated zero-mean white noise processes, $\left\langle\xi_{i}(t)\right\rangle=0,\left\langle\xi_{i}(t) \xi_{j}\left(t^{\prime}\right)\right\rangle=2 D \delta_{i j} \delta\left(t-t^{\prime}\right)$. In the absence of time delay $(\tau=0)$ and for large $N$, as the coupling strength $K$ exceeds a critical threshold $K_{c}$, the model (1) shows drastically different transitions to collective synchronization, depending on the shape of $g(\omega)$ [1-3,7-9]. For a strictly unimodal distribution the transition occurs between a totally incoherent state and a partially synchronized state. In contrast, symmetric bimodal distributions give rise to hysteresis and/or a transition to a time-dependent state composed of two clusters $[8,9]$.

The interactions in ensembles of coupled oscillators have been traditionally considered to be instantaneous, an assumption that considerably simplifies the analysis of such systems. However, the study of phase oscillators with time-delayed coupling [21] is receiving interest since a number of theoretical studies show that time delay may affect the synchronization phenomena considerably, typically leading to multi-

\footnotetext{
*Present address: Instituto de Física de Cantabria (CSIC-UC), Santander, Spain.
}

stability of many synchronous states (see, e.g., [3,4] and references therein). In particular, the Kuramoto model with unimodal frequency distribution has been generalized to allow time-delayed interactions in $[6,10]$. Additionally, phase models with time delay have successfully explained synchronization between plasmodial oscillators [11] and in semiconductor laser arrays [12]. Recent studies also demonstrate that time delay may be a useful synchronization-control mechanism in large oscillatory populations [13].

In this paper, we investigate the effects of a bimodal frequency distribution on the Kuramoto model (1) with time delay $\tau$. For $\tau=0$ and assuming that $g(\omega)$ is symmetric (centered at $\bar{\omega}$ with twin peaks of width $\gamma$ at both sides), one may always transform to a rotating frame, such that the model (1) is symmetric under the reflection: $\left(\theta_{i}, \omega_{i}\right) \rightarrow\left(-\theta_{i},-\omega_{i}\right)$. Time delay generally breaks such symmetry, except for the specific values $\tau=\tau_{n} \equiv n \pi / \bar{\omega}(n \in \mathbb{N})$. Thus, at $\tau=\tau_{n}$ we expect to recover the typical reflection-symmetric stationary wave solutions found in the Kuramoto model with bimodal frequency distribution but without delay [9]. For general $\tau$ values, the breaking of the reflection symmetry should give rise to the structures already observed for models without time delay but with either asymmetric bimodal frequency distributions [14] or asymmetric coupling functions [15].

\section{FULLY SYNCHRONIZED STATES}

We begin our analysis by considering the noise-free case, $D=0$, and a frequency distribution that consists of two infinitely sharp peaks (i.e., $\gamma=0$ ),

$$
g(\omega)=\left[\delta\left(\omega-\omega_{\mathrm{I}}\right)+\delta\left(\omega-\omega_{\mathrm{II}}\right)\right] / 2
$$

with $\omega_{\mathrm{I}}<\omega_{\mathrm{II}}$. Even for this simple choice, the results are illustrative and far from trivial.

Let us first investigate the existence and stability of synchronized solutions of Eq. (1) consisting of two clusters of identical oscillators that rotate uniformly with angular velocity $\Omega$ and phase difference $\beta$, 


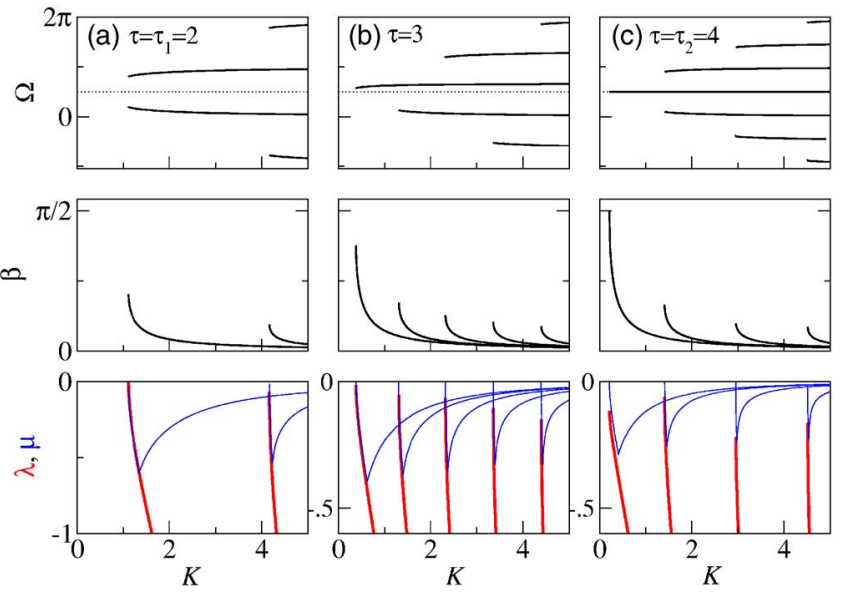

FIG. 1. (Color online) Frequency $\Omega$, phase difference $\beta$, and leading Lyapunov exponents $\lambda$ (thick red line), $\mu$ (thin blue line), for the stable synchronized solutions (3) as a function of $K$ for $\Delta \omega=\pi / 15, \bar{\omega}=\pi / 2$ (dotted line, upper panels).

$$
\Theta_{1,2}(t)=\Omega t \mp \beta / 2,
$$

with $\quad \Theta_{1} \equiv \theta_{j \leq N / 2}, \quad \Theta_{2} \equiv \theta_{j>N / 2} \quad$ (taking $\quad \omega_{j \leq N / 2}=\omega_{\mathrm{I}}$, $\omega_{j>N / 2}=\omega_{\text {II }}$. From Eqs. (3) and (1), we find two transcendental equations for $\Omega$ and $\beta$,

$$
\begin{gathered}
\Omega=\bar{\omega}-K \sin (\Omega \tau)[1+\cos \beta] / 2, \\
\sin \beta=\Delta \omega /[K \cos (\Omega \tau)],
\end{gathered}
$$

where $\Delta \omega \equiv \omega_{\text {II }}-\omega_{\text {I }}$ and $\bar{\omega} \equiv\left(\omega_{\text {I }}+\omega_{\text {II }}\right) / 2$. These equations have multiple solutions for given $K, \bar{\omega}, \Delta \omega$, and $\tau$ (see Fig. $1)$.

The linear stability analysis of the solutions (3) yields a pair of $\left(\frac{N}{2}-1\right)$-times degenerate eigenvalues,

$$
\lambda_{ \pm}=-K[\cos (\Omega \tau)+\cos (\Omega \tau \pm \beta)] / 2
$$

and a set of eigenvalues $\left\{\mu_{i}\right\}$ that are determined by the transcendental equation

$$
\left|\begin{array}{cc}
\left(1-e^{-\mu_{i} \tau}\right) c+c_{+}+\frac{2 \mu_{i}}{K} & -e^{-\mu_{i} \tau} c_{+} \\
-e^{-\mu_{i} \tau} c_{-} & \left(1-e^{-\mu_{i} \tau}\right) c+c_{-}+\frac{2 \mu_{i}}{K}
\end{array}\right|=0
$$

where $c_{ \pm} \equiv \cos (\Omega \tau \pm \beta)$ and $c \equiv \cos (\Omega \tau)$. The eigenvalues $\lambda_{+}, \lambda_{-}$govern the stability within the two individual clusters, each with $\frac{N}{2}$ oscillators. The eigenvalues $\mu_{i}$ (discarding the trivial solution $\mu_{0}=0$ ) are related to the stability of the frequency locking between the two clusters. Note that the results are independent of $N$, assuming it is even. We denote the leading Lyapunov exponents in each subset by $\lambda \equiv \max \left\{\lambda_{+}, \lambda_{-}\right\}$and $\mu \equiv \max \left\{\operatorname{Re}\left(\mu_{i}\right)\right\}(i \neq 0)$.

Figure 1 displays numerical solutions of Eqs. (4)-(7) for specific values of the parameters $\tau, \Delta \omega$, and $\bar{\omega}$. The upper panels show that for $\tau=\tau_{n} \equiv n \pi / \bar{\omega}$, symmetry-related solutions with frequencies $\Omega\left(K, \tau_{n}\right)=\bar{\omega} \pm \zeta\left(K, \tau_{n}\right)$ arise in pairs around the "central" frequency $\bar{\omega}$ (which is the synchroniza-
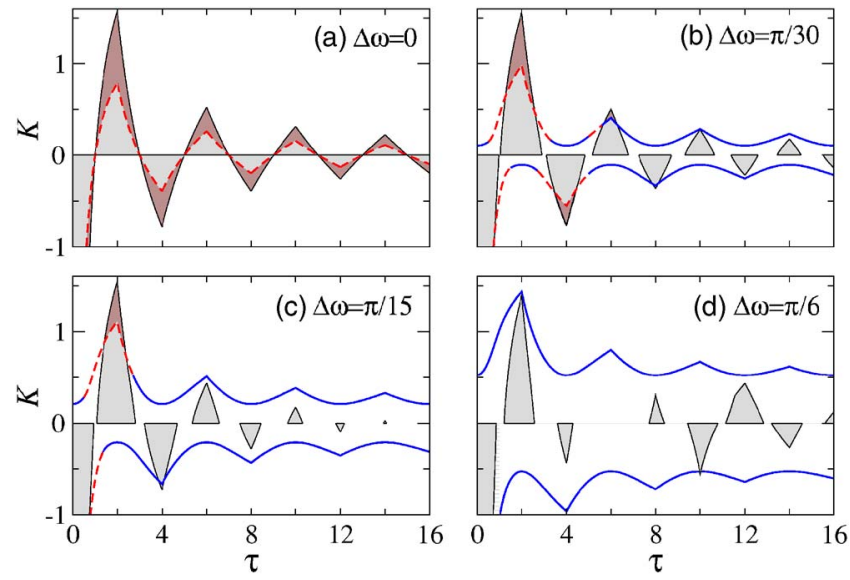

FIG. 2. (Color online) Stability boundaries for Synchronization and Incoherence. Theoretical boundaries for (full) synchronization are obtained from Eqs. (6) and (7). Disintegration $\left(\lambda \rightarrow 0^{-}\right)$and unlocking $\left(\mu \rightarrow 0^{-}\right)$are indicated with red-dashed and blue-solid lines, respectively. Black-thin curves: Boundaries of stable incoherence [Eq. (12)]. Shaded areas: Incoherence regions obtained by numerical integration of Eqs. (1), using a fifth-order Adams-Bashforth-Moulton scheme with $d t=\tau / 20$, except for $\tau$ $<1$, where $d t=0.05(N=24, \bar{\omega}=\pi / 2)$. Dark-shaded regions: bistability between incoherence and synchronization.

tion frequency for $\tau=0$ ) [Figs. 1(a) and 1(c)]. For positive $K$, the central solution is stable (unstable) for even (odd) values of $n$, and vice versa for negative $K$. For intermediate values, $\tau_{n}<\tau<\tau_{n+1}$, the stable solutions continuously vary between these two patterns, all of them approaching $\Omega=0$ as $\tau$ increases. This effect, common to time-delayed interacting oscillatory systems, is known as frequency suppression [16].

The growing number of synchronized solutions as $K$ and $\tau$ are increased (see Fig. 1) was already reported by Schuster and Wagner [17] for a system of two coupled oscillators, corresponding to model (1) for $N=2$ without the selfcoupling term. They also have shown that as $K$ is increased, stable solutions alternately appear with small $(\beta \approx 0)$ and large $(\beta \approx \pi)$ phase differences; this is also the case in the presence of self-coupling [22]. However, for a populationdue to the existence of the destabilizing modes linked to Eq. (6) - all the stable solutions are of the in-phase type, i.e., $0<\beta<\pi / 2$ (see Fig. 1, central panels). These solutions appear at saddle-node bifurcations where either $\lambda$ or $\mu$ vanishes (see Fig. 1, lower panels). Increasing $K$, the Lyapunov exponent $\lambda$ is stabilized $(\lambda \propto-K)$ whereas $\mu$ first decreases steeply and then abruptly changes its tendency (at the point where a pair of complex-conjugate eigenvalues $\mu_{i}$ become the leading ones), and asymptotically approaches zero.

Figure 2 shows the boundaries of the synchronous states (3) in the $\tau$ - $K$ plane, with incoherence regions obtained by numerical integration of Eqs. (1). For a unimodal distribution $(\Delta \omega=0)$, the regions without stable synchronous solutions are disconnected from each other, and the instability is always via the disintegration of the cluster [red dashed lines, Fig. 2(a)]. By contrast, for a bimodal distribution $(\Delta \omega>0)$ the stability boundaries detach from the $K=0$ axis and we obtain two separated continuous curves of marginal stability 
for $K<0$ and $K>0$. Note that as $\Delta \omega$ is increased, the instability of the phase-locked state occurs mostly via unlocking of the two clusters.

\section{INCOHERENT STATE}

Next we address an important type of solution of Eqs. (1), so-called incoherence, in which the system is in a completely phase-disordered state. To study the stability of the incoherent state, we introduce the complex order parameter $R e^{i \psi}=N^{-1} \sum_{j=1}^{N} e^{i \theta_{j}}$, which measures the degree of "phase coherence" in the system. This permits us to write the system (1) in terms of the time-delayed mean-field quantities $R$ and $\psi[1]$,

$$
\dot{\theta}_{i}(t)=\omega_{i}-K R(t-\tau) \sin \left[\theta_{i}(t)-\psi(t-\tau)\right]+\xi_{i}(t) .
$$

Considering the limit $N \rightarrow \infty$, we drop the indices and introduce the probability density for the phases $\rho(\theta, t, \omega)$ [18]. Then $\rho$ obeys the Fokker-Planck equation $\partial \rho / \partial t$ $=-\partial(\rho v) / \partial \theta+D \partial^{2} \rho / \partial \theta^{2} \quad$ (where $\quad v=\omega-K R(t-\tau) \sin [\theta(t)$ $-\psi(t-\tau)]$ ), for which the incoherent state $\rho_{0}=(2 \pi)^{-1}$ is always a trivial stationary solution. Linearizing the FokkerPlanck equation about $\rho_{0}$, one finds that the stability of incoherence is determined by the eigenvalues $\Lambda$ satisfying [6]

$$
e^{-\Lambda \tau} \frac{K}{2} \int_{-\infty}^{\infty} \frac{g(\omega)}{\Lambda+D+i \omega} d \omega=1 .
$$

\section{A. Bi-delta distribution without noise}

Equation (9) with the bi-delta distribution (2) and $D=0$ gives (after setting $\Lambda=-i \Omega_{c}$, to obtain the instability threshold) the collection of critical curves,

$$
\begin{gathered}
K_{c}^{(l)}=(-2)^{l}\left(\omega_{\mathrm{I}}-\Omega_{c}^{(l)}\right)\left(\omega_{\mathrm{II}}-\Omega_{c}^{(l)}\right) /\left(\bar{\omega}-\Omega_{c}^{(l)}\right), \\
\Omega_{c}^{(l)}=(1 / 2+l) \pi / \tau
\end{gathered}
$$

( $l$ integer), and two trivial ones $K_{c}=0$ (for $\Omega_{c}=\omega_{\mathrm{I}}$ and $\omega_{\mathrm{II}}$ ). This leads us to the bounds for stable incoherence,

$$
\begin{aligned}
& K_{c}^{+}(\tau)=\min \left\{K_{c}^{(l)}(\tau) \mid K_{c}^{(l)}(\tau)>0\right\}, \\
& K_{c}^{-}(\tau)=\max \left\{K_{c}^{(l)}(\tau) \mid K_{c}^{(l)}(\tau)<0\right\} .
\end{aligned}
$$

Figure 2(a) shows the regions of incoherence obtained from Eq. (12) for a unimodal distribution $(\Delta \omega=0)$ [6]. They consist of a periodic disposition of disconnected tent-shaped regions centered at $\tau=\tau_{n}$, of height $K_{c}=(-1)^{n+1} \pi / \tau_{n}$ and width $\tau_{1}$ (at $K=0$ ), as can be seen by setting $\bar{\omega}=\omega_{\mathrm{I}}=\omega_{\mathrm{II}}$ in Eq. (10).

In contrast-when $\omega_{\mathrm{I}}$ and $\omega_{\mathrm{II}}$ are incommensurate- the regions of incoherence for the bimodal frequency distribution (2) are organized quasiperiodically, with a decaying envelope of period $2 \pi / \Delta \omega$. Notice that this envelope becomes evident when $\Delta \omega$ is not small, as can be seen comparing Figs. 2(b) and 2(c) with Fig. 2(d).

This can be understood noticing that, for $|K| \approx 0$, such regions correspond to the overlapping of the incoherence regions of two independent unimodal populations (with
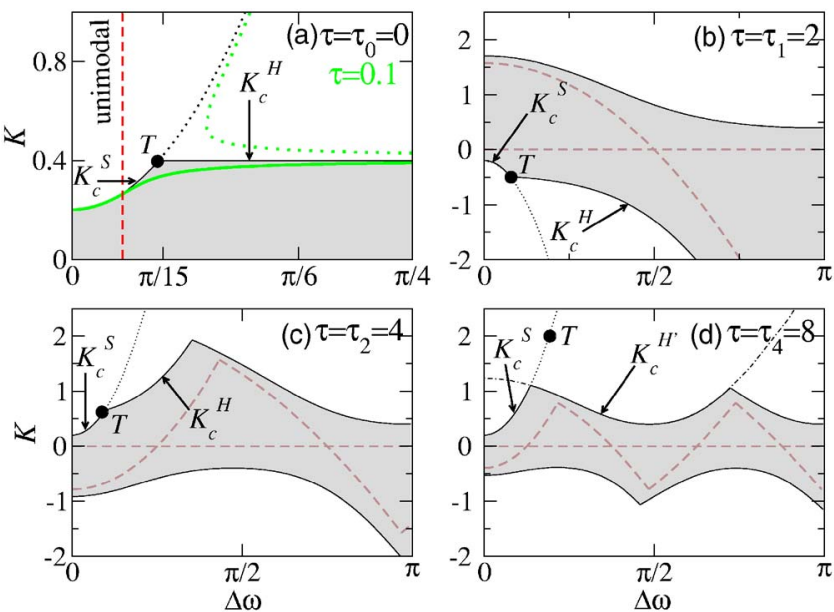

FIG. 3. (Color online) $\Delta \omega-K$ diagram showing the regions of stable incoherence for a bi-Lorentzian frequency distribution centered at $\bar{\omega}=\pi / 2$ and $\Gamma=0.1$. Solid-black $(\Gamma=0.1)$ and brown-dashed $(\Gamma=0)$ curves: analytical boundaries obtained from Eqs. (15) and (12), respectively. Shaded areas: Regions of incoherence obtained by numerical integration of Eqs. (1), $\gamma=0.1, D=0, N=2000$. (a) Green curves $(\tau=0.1)$ : symmetry breaking that results in two Hopf bifurcations (the solid green curve becomes the new boundary). To the right of the red-dashed line at $\Delta \omega=2 \gamma / \sqrt{3}$ [only shown in panel (a)], the bi-Lorentzian distribution is bimodal.

$\bar{\omega}=\omega_{\mathrm{I}}$ and $\left.\omega_{\mathrm{II}}\right)$. Thus, the new disposition of the regions of incoherence is (quasi)periodic with frequencies $\omega_{\mathrm{I}}$ and $\omega_{\mathrm{II}}$, and it can be determined through a function

$$
f(\tau)=\cos \left(\omega_{\mathrm{I}} \tau\right) \cos \left(\omega_{\mathrm{II}} \tau\right) \propto \cos (2 \bar{\omega} \tau)+\cos (\Delta \omega \tau),
$$

which takes positive values exactly where incoherence is stable for some $K \neq 0$.

\section{B. Presence of frequency diversity and noise}

For a unimodal frequency distribution, the inclusion of diversity (or noise) does not alter significantly the scenario already captured for identical oscillators [6], but for the bimodal distribution it has a more intricate effect.

We restrict our analysis to the bi-Lorentzian distribution, but a qualitatively similar scenario is expected for other bimodal distributions. Specifically, we take

$$
g(\omega)=\frac{\gamma}{2 \pi}\left(\frac{1}{\left(\omega-\omega_{\mathrm{I}}\right)^{2}+\gamma^{2}}+\frac{1}{\left(\omega-\omega_{\mathrm{II}}\right)^{2}+\gamma^{2}}\right),
$$

which, for $\Delta \omega>2 \gamma / \sqrt{3}$, becomes bimodal. Then the linear stability of the incoherent state, determined via Eq. (9), yields the transcendental equation

$$
\Lambda_{ \pm}=-\Gamma+\frac{1}{4}\left[K e^{-\Lambda_{ \pm} \tau} \pm \sqrt{K^{2} e^{-2 \Lambda_{ \pm} \tau}-4 \Delta \omega^{2}}\right]-i \bar{\omega},
$$

where $\Gamma \equiv D+\gamma$, and therefore, in what concerns the stability boundary, the effects of a frequency distribution and noise can be studied together.

With the help of Fig. 3(a), let us first recall the main results for $\tau=0$ [9]. For small separation of the peaks, 
incoherence becomes unstable in a steady-state bifurcation at $K_{c}^{S}=2\left[(\Delta \omega / 2)^{2}+\Gamma^{2}\right] / \Gamma$ (subcritically in the range $\left.2 \Gamma[(\Gamma+D) /(3 \Gamma+D)]^{1 / 2}<\Delta \omega<2 \Gamma\right)$. In this case, the system exhibits a single cluster of oscillators locked to the frequency $\Omega_{c}=\bar{\omega}$. If $\Delta \omega>2 \Gamma$, a degenerate Hopf bifurcation at $K_{c}^{H}=4 \Gamma$ precedes the previous instability. At $K_{c}^{H}$, two symmetric clusters at both sides of the central frequency $\bar{\omega}$ appear simultaneously. The loci of both bifurcations coalesce at $T$ (a double-zero eigenvalue point in the corotating frame).

A similar scenario is recovered periodically at the values $\tau=\tau_{n}$, i.e., when system (1) has reflection symmetry. The steady-state bifurcation occurs at the same values of $K$, but with a change of sign for odd $n$,

$$
K_{c}^{S}\left(\tau=\tau_{n}\right)=(-1)^{n} 2\left[(\Delta \omega / 2)^{2}+\Gamma^{2}\right] / \Gamma .
$$

Beyond the codimension-2 point $T$, located on the curve $K_{c}^{S}$, at $\Delta \omega=2 \Gamma \sqrt{\left(1+\Gamma \tau_{n}\right) /\left(1-\Gamma \tau_{n}\right)}$, this instability is preceded by a degenerate Hopf bifurcation [see Figs. 3(b) and 3(c)]. The latter expression diverges at $\tau=\Gamma^{-1}$ and hence, above a certain value of $n$ ( $n \geq 4$ for the parameters in Fig. 3), another degenerate Hopf bifurcation, denoted by $K_{c}^{H^{\prime}}$ in Fig. 3(d) (dash-dotted line), crosses the $K_{c}^{S}$ line and prevents incoherence to be stable for larger $K$ [23].

A general value of the time delay, $\tau \neq \tau_{n}$, changes this scenario due to the breakdown of reflection symmetry. For $\tau$ close to $\tau_{n}$, the Hopf bifurcations continuously split, as is shown in Fig. 3(a) for $\tau=0.1$. This occurs in a similar way as in previous works studying the breakdown of reflection symmetry without time delay $[14,15]$. Generally, such symmetry breaking implies asymmetric nonsimultaneous nucleations, as well as a complete modification of the bistability types and transitions to synchronization.

Figure 4 shows the region of incoherence in the $\tau$ - $K$ plane for two values of $\Delta \omega$. Due to the splitting of the degenerate Hopf bifurcations as $\tau$ is shifted from $\tau_{n}$, local extrema of incoherence appear exactly at $\tau=\tau_{n}$. Actually, the overall picture is more complex for arbitrary values of $\Gamma$, since some peaks are washed out, and different bimodal distributions may show specific shapes. However, in the $\Gamma \rightarrow 0$ limit, we provide a general distribution-independent statement [suggested from numerical solutions of Eq. (15) and analytical arguments]: peaks will appear at all $\tau=\tau_{n}$ values, unless there is a resonance $(\Delta \omega / \bar{\omega}=p / q)$ that excludes $n=2 m q / p, m$ even (odd) for positive (negative) $K$ values.

\section{CONCLUSIONS}

We have studied the Kuramoto model with time delay by analyzing the linear stability properties of the synchronized and incoherent solutions for bi-delta distributions (2). Our results have been compared to previous studies on phase oscillators coupled via time-delayed interactions. In particular,

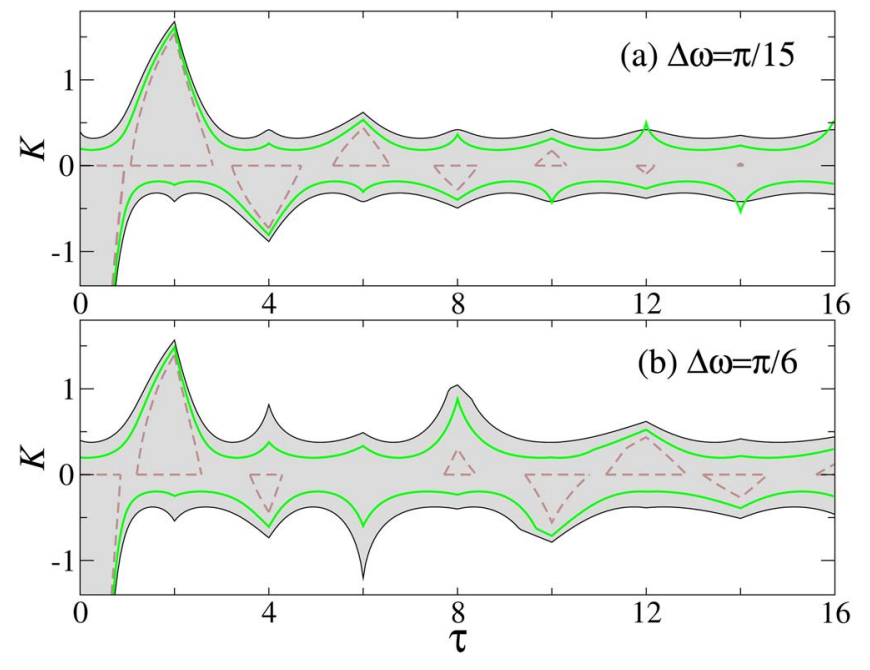

FIG. 4. (Color online) $\tau$ - $K$ diagram with the stable regions of the incoherent state for the bi-Lorentzian frequency distribution for $\Gamma=0$ [dashed brown, from Eq. (15)] and [from Eq. (12)] for $\Gamma=0.05$ (solid green) and $\Gamma=0.1$ (solid black). Shaded area: Incoherence regions obtained by numerical integration of Eq. (1) $(\gamma=0.1, D=0, N=2000)$.

in contrast to the $N=2$ case [17], the synchronous states only admit small phase differences. Also, compared to unimodal distributions [6], stability boundaries of the incoherent state have a completely different structure in parameter space, due to the presence of a new time scale in the system that results in a quasiperiodic pattern. Finally, we have considered the effect of diversity and/or noise on the incoherent state exploiting the reflection symmetry of the Kuramoto model for certain values of the time delay. For such values, we have achieved some analytical results that extend the nondelayed case studied in [9], and that help to build up a general picture for the shape of the regions of incoherence. These results may be of interest for the study of dynamical networks with bimodal frequency distribution, where the transmission times may play an important role in the synchronization dynamics, e.g., in neuroscience. For example, in the visual cortex, different populations of neurons form spatially separated cortical columns that interact with a significant time delay. In this context, a successful phase model for visual processing was proposed in [19], but the time-delay effects were only studied for two oscillators.

\section{ACKNOWLEDGMENTS}

We thank Claudio Tessone, Bernd Blasius, Raúl Vicente, and Jürgen Kurths for fruitful discussions. E. M. was partially supported by the European research project EmCAP (FP6-IST, Contract No. 013123). J. S. was supported by Deutsche Forschungsgemeinschaft project SCH-1642/1-1. 
[1] Y. Kuramoto, Chemical Oscillations, Waves, and Turbulence (Springer-Verlag, Berlin, 1984).

[2] S. H. Strogatz, Physica D 143, 1 (2000).

[3] S. C. Manrubia, S. S. Mikhailov, and D. H. Zanette, Emergence of Dynamical Order (World Scientific, Singapore, 2004).

[4] J. A. Acebrón et al., Rev. Mod. Phys. 77, 137 (2005).

[5] A. Winfree, J. Theor. Biol. 16, 15 (1967).

[6] M. K. Stephen Yeung and S. H. Strogatz, Phys. Rev. Lett. 82, 648 (1999).

[7] D. Pazó, Phys. Rev. E 72, 046211 (2005).

[8] L. L. Bonilla, J. C. Neu, and R. Spigler, J. Stat. Phys. 67, 313 (1992); H. Okuda and Y. Kuramoto, Prog. Theor. Phys. 86, 1159 (1991).

[9] J. D. Crawford, J. Stat. Phys. 74, 1047 (1994).

[10] M. Y. Choi, H. J. Kim, D. Kim, and H. Hong, Phys. Rev. E 61, 371 (2000).

[11] A. Takamatsu, T. Fujii, and I. Endo, Phys. Rev. Lett. 85, 2026 (2000)

[12] G. Kozyreff, A. G. Vladimirov, and P. Mandel, Phys. Rev. Lett. 85, 3809 (2000); A. G. Vladimirov, G. Kozyreff, and P. Mandel, Europhys. Lett. 61, 613 (2003).

[13] M. G. Rosenblum and A. S. Pikovsky, Phys. Rev. Lett. 92,
114102 (2004); O. V. Popovych, C. Hauptmann, and P. A. Tass, ibid. 94, 164102 (2005).

[14] J. A. Acebrón, L. L. Bonilla, S. DeLeo, and R. Spigler, Phys. Rev. E 57, 5287 (1998).

[15] E. Montbrió, J. Kurths, and B. Blasius, Phys. Rev. E 70, 056125 (2004).

[16] E. Niebur, H. G. Schuster, and D. M. Kammen, Phys. Rev. Lett. 67, 2753 (1991).

[17] H. G. Schuster and P. Wagner, Prog. Theor. Phys. 81, 939 (1989).

[18] S. H. Strogatz and R. E. Mirollo, J. Stat. Phys. 63, 613 (1991).

[19] H. Sompolinsky, D. Golomb, and D. Kleinfeld, Phys. Rev. A 43, 6990 (1991).

[20] E. M. Izhikevich, Phys. Rev. E 58, 905 (1998).

[21] A mathematically rigorous reduction from limit cycles with delayed interactions to phase oscillators is not fully justified [20], unless the time delay is considerably larger than the typical oscillation period. Nonetheless, important qualitative information is obtained considering both short and long delays altogether.

[22] B. Lysyansky (private communication).

[23] The bifurcation $K_{c}^{H^{\prime}}$ is not contained in the center manifold of $T\left(\Omega_{c} \mapsto \bar{\omega}\right.$ as $K \rightarrow K_{c}^{S}$ along $\left.K_{c}^{H^{\prime}}\right)$. 